\title{
PASADO Y PRESENTE EN RICOEUR Y DE CERTEAU. ALGUNAS CONSIDERACIONES.
}

\section{Esteban Lythgoe*}

Es fácilmente corroborable la línea general de la hipótesis de François Dosse según la cual la relación entre Paul Ricoeur y Michel de Certeau se fue consolidando a lo largo del tiempo ${ }^{1}$. Al respecto, se puede comparar la cantidad y el tipo de referencias que el filósofo hace de su colega en Tiempo $y$ Narración con las que realizará veinte años más tarde en La memoria, la bistoria $y$ el olvido. En el tercer tomo de Tiempo y Narración Michel de Certeau es mencionado en dos oportunidades. En ellas, el filósofo no sólo destaca la importancia de haber puesto de manifiesto el carácter definitorio que tiene la narrativa para la historia, sino que también reconoce su crítica a las visiones totalizantes de la historia, al pasado sustancial y a la idea de representación como reduplicación mental de la presencia. Sin embargo, tras los elogios toma distancia respecto de la concepción del pasado como desviación pues lo deja, dice, como un enigma irresoluble. La incidencia del historiador aumenta en La memoria, la historia y el olvido, como lo demuestra el uso de la expresión certeana de operación bistórica para caracterizar la complejidad de la labor del historiador, el reconocimiento de la inserción social del investigador y la ruptura que impone la escritura respecto del vínculo con el pasado.

En el presente artículo querríamos detenernos en dos aspectos de esta relación. La primera se dirige a señalar aquellos factores en el pensamiento del filósofo que facilitaron el acercamiento a Certeau. Nuestra

* Licenciado y doctor en filosofía por la Universidad de Buenos Aires. Actualmente es docente en la Universidad Argentina de la Empresa e investigador del Consejo Nacional de Investigaciones Científicas y Técnicas. Su temática de investigación está ligada al papel del testimonio de sobrevivientes de acontecimientos traumáticos y su rol de articulador entre la memoria colectiva y la historia. Dirección electrónica: lythgoe@arnet.com.ar

1 Dosse, François, Paul Ricoeur, Michel de Certeau. L'bistoire: entre le dire et le faire, L'Herne, Paris, 2006, p. 8. 
tesis es que lo que Ricoeur ha denominado la vuelta al acontecimiento ${ }^{2}$ ha sido decisivo para este acercamiento en al menos dos aspectos: por una parte, el problema de la relación entre narración histórica y pasado; por otra, la apertura a consideraciones de la memoria, sus patologías y, consecuentemente, la incorporación del psicoanálisis a las preocupaciones históricas. El segundo aspecto en el que nos detendremos son las críticas que Ricoeur le realizara en Tiempo y Narración con respecto a la cesura que el historiador plantea entre la representación histórica y el pasado y al hecho de no considerar como vivas las posibilidades beredadas. Evaluaremos su pertinencia y discutiremos en qué medida pueden afectar a los propios planteos de Ricoeur tal como los desarrolla en La memoria, la bistoria y el olvido.

1.

En el primer tomo de Tiempo y Narración Ricoeur presenta su teoría narrativa de la historia basada en los conceptos de muthos y mimesis. Uno de los puntos centrales de este análisis es su debate con la escuela de los Annales y la epistemología analítica por su rechazo al uso de los conceptos de acontecimiento y narración en historia.

Si bien la epistemología analítica utiliza el término "acontecimiento" en historia, su sentido es diferente al de la historia. En su artículo "La función de las leyes generales en historia", Carl Hempel subordina al acontecimiento histórico bajo el concepto general de acontecimiento, junto también con el acontecimiento físico. De este modo, el historiador debería subordinar el acontecimiento a una hipótesis universal tal como cualquier otro científico. Rápidamente se puso de manifiesto que este modelo explicativo no era representativo de la labor del historiador, pues éste destaca la singularidad del acontecimiento por sobre lo que comparte con otros similares.

Por su parte, la escuela de los Annales se enfrentó en sus inicios a la tradición positivista francesa de la primera parte del siglo $\mathrm{XX}$ que se apoyaba en los acontecimientos. Los Annales disintieron con tres aspectos

${ }^{2}$ Cfr. Ricoeur, Paul, «Événement et sens » en Raisons pratiques 2, 1991, p. 41. 
de este uso: que su iniciador deba ser un actor individual identificable, que sea una oscilación breve y explosiva y que su modo de trasmisión sea la narración. Su crítica de la historia de los acontecimientos, por lo tanto, se dirigió contra estos supuestos. Así, tematizaron el 'hecho social total' en todas sus dimensiones, enfatizando la larga duración y recurriendo a métodos cuantitativos.

Para responderle a esta escuela, Ricoeur mantiene la asociación entre narración y acontecimiento por ellos planteada, pero, siguiendo a Aron y Marrou, pone en duda que el acontecimiento sea aquello que efectivamente se produjo en el pasado. Los historiadores no tienen un contacto directo con el pasado, sino que sólo acceden a reconstrucciones ${ }^{3}$. El pasado realmente vivido por la humanidad está fuera de su alcance, sólo puede ser postulado cual noúmeno kantiano en el origen del fenómeno empírico conocido. El acontecimiento no prima por sobre la intriga, sino que es un emergente de ella.

En consecuencia, un acontecimiento debe ser más que una ocurrencia singular. Recibe su definición de su contribución al desarrollo de la intriga. Una historia, por otra parte, es más que una enumeración de acontecimientos en un orden serial, debe organizarlos en una totalidad inteligible, de tal manera que podamos siempre preguntarnos cuál es el tema de la historia ${ }^{4}$.

En el debate con Braudel es aún más explícito al afirmar que "el acontecimiento es una variable de la intriga"5. Con "acontecimiento" Ricoeur está haciendo referencia a lo que dos décadas más tarde denominará becho, es decir, el constructo lingüístico referido a una realidad pasada. Mientras que el acontecimiento narrativo es un emergente derivado de esta construcción proposicional, el acontecimiento bistórico es postulado a partir del anterior. Así se explica:

${ }^{3}$ Cfr. Ricoeur, Paul, Temps et Récit III (TRIII), Paris, Seuil, 1985, p. 175.

4 TRIII, p. 127.

${ }^{5}$ Ricoeur, Paul, Temps et Récit I (TRI), Paris, Seuil, 1983, p. 383. 
la derivación indirecta de las estructuras de la historiografía a partir de las estructuras de base del relato, establecida en las secciones precedentes, permite pensar que es posible, por procedimientos apropiados de derivación, extender a la noción de acontecimiento bistórico la reformulación que la noción de acontecimiento-puesto-en-intriga ha impuesto a los conceptos de singularidad, de contingencia y de desviación absolutos ${ }^{6}$.

Para el filósofo francés habría un punto de contacto entre narración histórica y pasado, pero éste no se produciría a nivel del acontecimiento, sino de la puesta en intriga. En efecto,

sea cual fuera la fuerza de innovación de la composición poética en el campo de nuestra experiencia temporal, la composición de la intriga está enraizada en una pre-comprensión del mundo de la acción: de sus estructuras inteligibles, de sus recursos simbólicos y de su carácter temporal ${ }^{7}$.

Esta raíz común entre el mundo de la acción y el mundo de la narración no conduce a una identificación de la narración histórica con el pasado, como lo hiciera oportunamente Collingwood. En opinión de Ricoeur, esta identificación no rinde cuenta de la alteridad de la narración histórica. En contraposición, acepta sostener que narración y pasado son dos instancias diferentes entre sí como lo sostienen el narrativismo y Paul Veyne, entre otros. Sobre esto último arguye que al explicar la historia por invariantes, los acontecimientos históricos han sido destemporalizados haciendo desaparecer el atributo definitorio de la historia. Frente a estas dos alternativas el filósofo francés propone a la representancia como un vínculo analógico entre el pasado y su narración. La narración toma el lugar del pasado y hace como si fuera él, gracias a lo cual "retiene en sí la fuerza de la reefectuación y de la puesta en distancia, en la medida en que ser como, es ser y no ser"8.

${ }^{6}$ TRI, p. 365.

7 TRI, p. 108.

8 TRIII, p. 226. 
2.

Ricoeur le hace dos objeciones a de Certeau. La primera está ligada con el tipo de vínculo que existe entre la escritura histórica y el pasado; la segunda, con el tipo de posibilidades heredadas del pasado. Con respecto a la primera crítica, Ricoeur ubica a su colega dentro del grupo que concibe al pasado como diferente de la narración. Si bien reconoce su importancia como crítica a las concepciones totalizantes de la historia y un exorcismo a la idea de pasado sustancial, le objeta la ausencia de una caracterización positiva del pasado. Así, "lo real del pasado resta el enigma del que la noción de diferencia-desviación, resultante del trabajo sobre el límite, no ofrece sino una suerte de negativo, despojado además de su perspectiva propiamente temporal"9. La segunda objeción, por su parte, surge a partir de la comparación entre los conceptos de deuda utilizados. Ricoeur señala la existencia de cierto parentesco entre su concepto de deuda y el utilizado por de Certeau en La escritura de la bistoria, especialmente en el capítulo dedicado al análisis de Moisés y el monoteísmo de Freud. Este último, con todo, recoge principalmente el componente negativo ligado con la obligación (la desposesión y el exilio). En palabras de Ricoeur:

Al ligar así la deuda a la pérdida, M. de Certeau pone más el acento sobre la "tradición de un muerto", pero no señala suficientemente a mi parecer el carácter positivo de la vida babiendo sido, en virtud del cual la vida es también herencia de potencialidades vivas" 10 .

Coincidimos hasta cierto punto con Ricoeur respecto de esta última observación. De Certeau considera que tanto el psicoanálisis como la historia giran en torno a la relación entre el presente y el pasado, dejando de lado la cuestión del advenir que, como veremos más adelante, es donde se apoya Ricoeur al referirse a la cuestión de las posibilidades. Para el historiador francés la historia y el psicoanálisis deben buscar cada uno a su manera: a) establecer criterios para comprender las diferencias $y$ continuidades entre el presente y el pasado; b) hacer que el presente pueda

9 TR III, p. 218.

10 TR III, p. 227, nota 1. 
explicar el pasado; c) traer las representaciones a sus condiciones de producción; y d) elaborar maneras de pensar y superar la violencia de los conflictos de la historia ${ }^{11}$. Más allá de que Certeau está concibiendo a la historia de un modo distinto al de Ricoeur, a punto tal que es discutible la pertinencia del calificativo de "crítica", es posible objetarla apoyándose en el último de estos objetivos. Es decir, en su carácter crítico y superador del pasado sería posible reconocer una suerte de vitalización y reiteración de ciertas posibilidades conciliadoras sidas. Andris Breitling pone un límite de todas maneras a esta última afirmación al sostener que si bien el componente psicoanalítico de Certeau conlleva un elemento liberador, "con todo, en la recurrencia de temas tales como la pérdida, la 'desposesión' y el 'exilio' se manifiesta un primado de lo negativo que continúa en Certeau hasta en las formulaciones en apariencia afirmativas"12.

Pero más interesante nos resulta aquí la crítica de Ricoeur respecto de la caracterización del pasado. Para de Certeau, la relación entre pasado y presente de la práctica historiográfica se debe en gran medida a los soportes técnicos (archivos, talleres, etc.). Tradicionalmente, la práctica historiográfica partía del número limitado de restos que se poseía en ese momento y los unificaba en una comprensión coherente. Si aparecían nuevos trazos que afectaran esta comprensión, ésta debía ser replanteada. Con la aparición de la computación el proceso acaba siendo invertido: se parte construyendo un modelo cuyo objetivo es encontrar la desviación, el 'error' o lo heterogéneo de la combinación de varias series. El pasado se constituye como lo otro del presente al verse como la diferencia constituida en el límite de los modelos. Así, el pasado 'nombra lo incomprendido'13 y en este sentido Ricoeur sostiene que Certeau hace una caracterización negativa del pasado. Paradójicamente, el objetivo de la historia moderna no

${ }^{11}$ Cfr. de Certeau, Michel, Historia y Psicoanálisis entre ciencia y ficción (HP), Universidad Iberoamericana, México, 1995, p. 79.

12 Breitling, Andris, "L'écriture de l'histoire: un acte de sépulture ?", en Myriam Revault d'Allones et François Azouvi (dir.), Paul Ricoeur II, L'Herne, Paris, 2004, p. 125.

${ }^{13}$ Cfr. de Certeau, Michel, L'écriture de l'bistoire (EH), Gallimard, Paris, 1975, p. 119. 
consistiría en explotar lo diferente sino en anular la diferencia, para volverlo comprensible por medio de categorías contemporáneas ${ }^{14}$.

El escrito histórico se encontraría entonces en una situación ambivalente: su retórica apunta hacia lo otro del pasado, pero sus prácticas han eliminado esa alteridad.

Con todo, el modo en que el historiador distingue entre acontecimiento y hecho obliga a matizar la objeción ricoeuriana, pues se observa que la cesura sólo se produce a nivel gnoseológico y no ontológico. En efecto, los hechos son una organización de sentido, enunciados por medio de un lenguaje referencial. Por su parte, el acontecimiento es aquello que no se comprende, el límite de la historia. Es el punto de partida postulado de la comprensión que no explica, pero que permite una inteligibilidad. Sin embargo, el acontecimiento no es algo que nos llega pasivamente sino que está constituido desde el presente. No es posible definir a priori un acontecimiento histórico, pues éste es tal en la medida en que modifica la historia. En efecto, "uno de los criterios para poder emitir un juicio consiste en los cambios epistemológicos que provoca. En pocas palabras, lo que hacemos del acontecimiento sólo permite calificarlo de acontecimiento"15. Desde el punto de vista de la construcción de la historia, ambas instancias son necesarias y recíprocas entre sí: "el acontecimiento es aquello que recorta, para que haya inteligibilidad; el hecho histórico es aquello que plenifica para que haya enunciados de sentidos"16.

Este matiz es reforzado aún más si tenemos en cuenta el abordaje alternativo que puede hacer el psicoanálisis al pasado. Aún cuando psicoanálisis e historia compartan sus preguntas fundamentales, conciben de

14 “Pues la misión que le asigna el más allá (el por acá) del presente tiene, precisamente, como objetivo traer al otro al campo de una comprensión presente y, en consecuencia, eliminar la alteridad que parecía ser el postulado de la misión. Lo otro no sería la condición de posibilidad, mantenida externa, del discurso filosófico, sino lo contrario: transformado en objeto, el elemento que el discurso histórico transforma en significantes y reduce a algo inteligible para eliminar este peligro" (de Certeau, Michel, Historia y Psicoanálisis entre ciencia y ficción. Nueva Edición (HP2), Universidad Iberoamericana, México, 2002, p. 117).

${ }^{15} \mathrm{HP} 2$, p. 113.

${ }^{16} \mathrm{EH}, \mathrm{p} 134$. 
manera diferente la relación entre el presente y el pasado: en el primero hay imbricación y en el segundo sucesión. En el psicoanálisis el pasado no es una alteridad inaccesible, sino que se lo puede reconstruir. El escrito histórico es una homogeneidad en la que se ha eliminado la alteridad del pasado. Al interpretar el pasado de múltiples maneras, el historiador no hace sino ocultar el pasado simple que se debía develar. "Las explicaciones efectúan un despliegue de contrarios; por ello, multiplican las representaciones, es decir rompen el Urbild en mil facetas cuando ellas lo repiten en un lenguaje 'analítico"'17. Certeau compara este proceso con lo que sucede con un enfermo cuando ingresa a un hospital psiquiátrico: sus síntomas se esfuman en el cuerpo social del saber psiquiátrico. El analista se encuentra en condiciones de reinstaurar la heteronomía, reorganizando las palabras en función de cosas borradas o perdidas. Esta historia-trabajo le permite acceder nuevamente al conflicto, ya no en calidad de imagen sino a título de la ley que organiza cada nuevo lenguaje. Teniendo en cuenta que estas consideraciones no son ajenas a Ricoeur resulta extraña su crítica ${ }^{18}$.

A continuación, querría reformular la crítica realizada por Ricoeur a la luz de sus desarrollos en La memoria, la bistoria, el olvido, pues nos abrirán nuevas perspectivas acerca de la relación entre el pasado y el presente y el lugar que en ella le cabe a la psicología.

17 “[E]l psicoanálisis trata esta relación bajo el modo de la imbricación (uno en el lugar del otro), de la repetición (uno reproduce al otro bajo otra forma) del equívoco y la equivocación [...] La historiografía considera esta relación bajo el modo de la sucesión (uno después de otro), de la correlación (proximidades más o menos grandes), del efecto (uno sigue al otro) y de la disyunción (o uno o el otro, pero nunca los dos a la vez)." (EH, p. 353).

${ }_{18}$ Cfr. TR III. 227 Nota 1. Todas estas consideraciones me acercan a F. Dosse quien afirma que "ciertas lecturas de $\mathrm{M}$. de Certeau han tenido la tendencia de ver en él uno de los representantes en Francia del Giro Lingüistico y a encerrarlo en un acercamiento puramente retórico del discurso histórico al interior de una concepción exclusivamente discursiva de la historia. [...] Bien por el contrario, él insiste sobre la apertura por la historia de un espacio inédito alrededor de la búsqueda de la verdad que la distingue fundamentalmente del simple 'efecto de real' según los términos de Roland Barthes" (F. Dosse, op. cit., p. 177). 


\section{3.}

La memoria, la historia, el olvido se ubica en un contexto historiográfico diferente. Diez años antes de su publicación se produjo lo que Delacroix denominó giro crítico $^{19}$, giro que supuso el abandono del método cuantitativo y la larga duración. La historia abandona su interés por los procesos supraindividuales para retornar a la conciencia y a los individuos en calidad de iniciadores de la acción y varios autores. Haciendo eco de este giro, Ricoeur toma el acontecimiento y no las estructuras narrativas como la base de sus análisis. Esta modificación permite, en primer lugar, la incorporación de la memoria como punto de partida, algo impensable desde una historia basada en las estructuras narrativas, dado que lo que caracteriza a la memoria es su capacidad de fijar los acontecimientos pasados. Como lo define el filósofo, "se dice indistintamente que uno se representa un acontecimiento pasado o que uno tiene una imagen de él, que puede ser cuasi visual o auditiva" 20 . Junto con la memoria se incorporan también todas sus patologías y manipulaciones. Como veremos más adelante esta incorporación acercará la problemática del historiador a la del psicoanalista.

La segunda consecuencia de tomar el acontecimiento como instancia fundadora del análisis histórico, es que la diferencia entre el plano histórico y la realidad pasada será destacada por medio de la distinción entre becho histórico y acontecimiento. El primero es un constructo surgido de diversos procedimientos y apoyado en una serie de justificaciones. Su carácter es proposicional, y por tanto, verificable. El acontecimiento, en cambio, es el 'contenido de un enunciado que intenta representarlo'. Esto significa que, para ser precisos, tendríamos que decir: "el hecho de que esto aconteció". La memoria recoge los acontecimientos y los testigos los transmiten. Ninguna de las cosas trasmitidas es verdadera o falsa, porque entran en el ámbito de la atestación ${ }^{21}$.

19 Delacroix, Christian, "La falaise et le rivage. Du 'tournant critique' à la conversión pragmatique des Annales (1995) » en Espaces Temps. Les Cabiers, num. 5960-61, 1995, pp. 3-4.

${ }^{20}$ Ricoeur, Paul. La mémoire, l'bistoire, Ioublie (MO), Seuil, Paris, 2000, p. 5.

${ }^{21}$ Ricoeur distingue los dos ámbitos del siguiente modo: "¿Y no propuse yo mismo al tratar del hecho histórico, distinguir la proposición que enuncia 'el hecho de 
La escritura se convierte así en la frontera entre la memoria y la historia como de dos regímenes dóxicos diferentes: la atestación, del lado de la memoria, y la episteme a nivel de la historia. Entre ambos se erige la figura del archivista: él es quien transcribe las declaraciones orales para preservarlas y clasificarlas, y, en su pasaje del medio oral al escrito, introduce el elemento crítico que permite asegurar de algún modo la objetividad de lo dicho. El archivista aplica toda herramienta crítica a su alcance para distinguir la declaración verdadera de la falsa a través del método indiciario. Al proceder según el ideal del paradigma de registro, el archivista preserva el carácter contrastable de las pruebas $y$, por lo tanto, la pretensión de verdad en historia. Este proceso permite normalizar al testimonio respecto de las demás fuentes de conocimiento histórico ${ }^{22}$.

Como se desprende de lo que hemos dicho, el uso de la distinción entre hecho y acontecimiento acercó el pensamiento de ambos pensadores tanto en lo que respecta a la relación entre la historia y el psicoanálisis como a la relación entre presente y pasado. Asimismo, tal acercamiento podría volver a Ricoeur pasible de las objeciones acerca de la diferencia entre pasado y presente que oportunamente le realizara a Certeau. Es por este motivo que querríamos concluir adentrándonos en el análisis que el filósofo hace de la condición histórica a fin de establecer si logra evitar esa crítica y de hacerlo de qué modo.

4.

La intención de Ricoeur es reelaborar las consideraciones heideggerianas en torno a la temporalidad, y, más específicamente, el

que...' del acontecimiento mismo? El realismo crítico profesado aquí es obligado a dar un paso más de este lado de la proposición factual e invocar la dimensión testimonial del documento. En efecto, la fuerza del testimonio se expone precisamente en el corazón mismo de la prueba documental" (MO, p. 364).

22 "Estos testimonios orales sólo constituyen documentos una vez registrados; dejan la esfera oral para entrar en la de la escritura, se alejan así de la función del testimonio en la conversación ordinaria. Se puede afirmar entonces que la memoria está archivada, documentada. Su objeto ha dejado de ser un recuerdo en el sentido propio del término, es decir, retenido en relación de continuidad y de apropiación respecto a un presente de la conciencia." (MO, p. 226). 
existenciario de la deuda como condición de posibilidad de la representancia, para utilizarla como base de su fundamentación ontológica de la epistemología de la historia. Este análisis abarcará los tres aspectos temporales tratados en Ser y tiempo: la temporalidad, la historicidad y la intratemporalidad.

Más que la temporalidad, Ricoeur se apoya en las consideraciones heideggerianas en torno a la muerte como punto de partida de esta articulación entre historia y filosofía. Según Heidegger, el precursor-estadode-resuelto es la condición de posibilidad para el acceso de la temporalidad como el sentido del ser del Dasein. El precursar-la-muerte nos hace tomar conciencia de nuestra finitud, reasumir nuestro vínculo con el pasado y hacernos cargo de ciertas posibilidades con las cuales enfrentar el presente. Esta estructura le permitirá descubrir a la temporalidad como tres éxtasis cooriginarios: advenir, sido y presente, donde el advenir prima por sobre los demás. Sin embargo, el costo de este acceso consiste en caer en una suerte de solipsismo. En efecto, el precursar-la-muerte heideggeriano supone comprenderse a sí mismo a partir de esta posibilidad irrebasable, oponiéndose a las concepciones cotidianas de la muerte, como plenitud, totalidad o cesar, surgidas de nuestra experiencia cotidiana.

En contraposición con esto, la historia nos remite constantemente a la muerte de los demás. Por lo tanto, el único modo de recurrir a una temporalidad fundada en un Dasein propio y total consiste en desdibujar la distinción entre lo propio y lo impropio, sacando a la analítica existenciaria de su encierro solipsista y restituyendo la densidad ontológica del se muere impropio 23 . Para lograr este objetivo Ricoeur reemplaza lo que denomina la vía corta heideggeriana, que establece un vínculo directo entre el poder-ser y la muerte, por otra larga, que abra al Dasein a la exterioridad y la factualidad. Logra la exterioridad el cuerpo propio y la carne en la analítica existenciaria. Esta incorporación nos permite reconocer que, desde el punto de vista genético, nuestro primer contacto con la muerte surge de la observación de cómo la muerte se presenta en la naturaleza a la manera de un obstáculo al deseo de vivir. El abismo que separa estas primeras experiencias respecto de la posición heideggeriana en la que el precursar-la-

${ }^{23}$ Cfr. MO, p. 475. 
muerte es parte de la comprensión del sí se resolvería gracias a un proceso de introyección. La factualidad, por su parte, nos remite al contacto con la muerte a través de mis semejantes y es constitutivo de este proceso de introyección. Mientras Heidegger sostiene que el contacto con la muerte a través de los demás es banalizante, para Ricoeur, por el contrario, enriquece la concepción que uno tiene de sí mismo, pues permite tomar conciencia de que frente a la muerte uno es igual a su prójimo.

De Certeau también considera que la muerte tiene un papel muy importante en la historia occidental, a punto tal que asocia a la escritura de la historia con el momento del entierro del muerto, en el que, por una parte, se exorciza a la muerte introduciéndola en el discurso, y por la otra, la función simbolizadora le proporciona al lector un pasado, asignándole de este modo un lugar a tomar y un deber ser a cumplir. En contraposición con Heidegger, que recurre a la muerte como vía de acceso a la temporalidad, el historiador francés desarrolla la problemática de la muerte fundándose en su concepción de la relación entre el presente y el pasado. A pesar de que esta insistencia por representar a los muertos pareciera contraponerse al trato negativo del pasado por parte de la práctica historiográfica, su fundamentación es consistente con lo desarrollado más arriba. En efecto, los vivos del presente entierran a sus muertos para tener así un lugar para vivir. Hablar de los muertos es una manera de exorcizarlos, de negarlos, y justamente la escritura en tanto entierro nos remite al pasado para honrarlo y eliminarlo ${ }^{24}$.

A pesar de coincidir con el papel fúnebre que de Certeau le asigna a la historia, Ricoeur lo defiende de otro modo. En su opinión, el entierro permite que algo de los muertos pueda mantenerse vivo y que los sucesores lo puedan aplicar en contextos diferentes.

Una meditación sobre la reiteración autoriza un paso más, al establecer la idea que los muertos de otra época han estado vivos y que la historia,

24 “[S]ustituto del ser ausente, encierro del genio maléfico de la muerte, el texto histórico cumple un rol performativo. El lenguaje permite a una práctica situarse respecto de su otro, el pasado. De hecho, él mismo es una práctica. La historiografía se sirve de la muerte para enunciar una ley del presente" (EH, p. 141). 
de una cierta manera, se acerca a su haber-estado-vivos. Los muertos de hoy son los vivos de ayer, actuantes y sufrientes ${ }^{25}$.

Esta afirmación consta de dos aspectos: cómo se vincula la historia con el pasado (la historia se acerca a su haber-estado-vivos) y el carácter de quienes vivieron en el pasado (los muertos de hoy son los vivos de ayer, actuantes y sufrientes). La primera de ellas será abordada en la historicidad, en tanto la segunda se abordará en la intratemporalidad.

Consideramos que Ricoeur cierra la distancia entre el acontecimiento y el hecho en la etapa de la historicidad al resolver el problema heideggeriano del vestigio. Heidegger plantea la existencia de dos regímenes de tiempos diferentes: mientras los objetos pasados ya no son, el Dasein ba sido. En el caso del vestigio sucede algo extraño porque nos encontramos con un objeto que tiene valor histórico. Esto se opone a lo recién señalado, pues en tanto ente-ante-los-ojos entra en el régimen del sido y no en el ya no ser. Tal carácter histórico no se debe a ningún atributo particular de la persistencia del tiempo en el vestigio, sino al vínculo del Dasein con el pasado a través de la deuda. Para articular ambos aspectos, el filósofo se apoya en la definición aporética de la representación donde se lo concibe como una imagen presente de una cosa que tuvo una existencia pasada, 'ha sido', pero que ya ha desaparecido, 'ya no es'. Estas dos expresiones son asociadas a los dos tipos de representación: la que corresponde a la memoria y la de la historia. Cuando se afirma que la representación mnémica ha sido significa que tuvo su origen en el pasado y sus efectos se mantienen a lo largo del tiempo. La representación histórica, en cambio, es una imagen de algo que ya no es. Lo que propone el filósofo es asignar diferentes prioridades a estos tipos de representación. Así afirma:

no es inaceptable sugerir que el 'haber sido' constituye el último referente al que se apunta a través del 'ya no ser'. La ausencia sería así desdoblada entre una ausencia como objetivo de la imagen presente y la

${ }^{25} \mathrm{MO}$, p. 495. 
ausencia de las cosas pasadas en tanto que pasadas respecto a su haber sido $^{26}$.

A fin de justificar esta última afirmación, Ricoeur recurre a los conceptos de generación y reiteración de Heidegger, al de resurrección de Michelet $\mathrm{y}$ al de re-efectuación de Collingwood. Mientras la generación destaca el carácter cultural y lingüístico de la transmisión hereditaria, la reiteración alude a un tipo de réplica o respuesta a la herencia que no busca restituir o repetir el pasado, sino realizar sus posibilidades nuevamente teniendo presente los proyectos actuales del Dasein. Michelet y Collingwood, por su parte, ponen de manifiesto la necesidad de distinguir la ocurrencia del acontecimiento pasado de su significación. La primera se encuentra ligada con el trazo en tanto objeto físico, una pura remisión al pasado del pasado, que no puede modificarse, pero tampoco nos obliga a nada. La significación del pasado o su componente de sentido, en cambio, sí puede modificarse y, en tanto ligado con la deuda, nos obliga y liga asimismo al futuro. En la medida en que los proyectos y posibilidades de quienes nos precedieron no tienen por qué quedar congelados en el pasado, se rompe con el determinismo histórico y reintroduce la contingencia histórica. Como corolario de este planteo, nos encontramos con lo que consideramos es una de las diferencias más importantes entre Ricoeur y Certeau y es que la representación histórica no debe estar dirigida solamente hacia el pasado sino también hacia el futuro.

A fines de la década de los ochenta la escuela de los Annales abandona su proyecto ligado a la larga duración y su interés por los largos ciclos y, en su lugar, reconoce el papel de los individuos como iniciadores generadores de cambio. Entre los aportes de este giro pragmático Ricoeur recoge el tratamiento que Bernard Lepetit hace del concepto de competencia como la capacidad del agente social para negociar los conflictos y del de ajuste entre la voluntad individual y la norma colectiva. En la última etapa del diálogo, Ricoeur confronta estos aportes con el tiempo de la preocupación de la analítica existenciaria. A diferencia de las instancias anteriores donde había una suerte de amoldamiento entre historia y filosofía, en este caso se plantea la existencia de una equivalencia entre

${ }^{26} \mathrm{MO}$, p. 367. 
ambos ámbitos. Así, se afirma "a este respecto, las nociones de competencia y de ajuste dicen el equivalente historiográfico de la preocupación heideggeriana"27. Aunque no lo afirme explícitamente, estas consideraciones parecen proporcionar elementos adicionales para rechazar la concepción certeana de la muerte y sostener que "el objetivo de la historia no sólo es el viviente de antaño, a la zaga del muerto de hoy, sino el actor de la historia pasada, puesto que se intenta 'tomar en serio a los actores mismos"'28.

5.

Hemos visto y comparado los modos en que Ricoeur y Certeau concebían la relación de la historia con el pasado. Ricoeur afirmaba en Tiempo y narración que la historia proporciona una suerte de análogo del pasado. En esa obra el filósofo le objetaba a de Certeau, por una parte, considerar que la escritura histórica no daba cuenta del pasado, y por la otra, no destacar el carácter vivo y reiterable que tienen las posibilidades sidas. En La memoria, la bistoria, el olvido, Ricoeur vuelve al concepto de acontecimiento, lo que estrechó el vínculo con de Certeau. Por una parte, las consideraciones mnémicas permitieron incorporar planteos psicoanalíticos a la historia. Por la otra, nos llevó a considerar si la distinción entre hecho y acontecimiento no abría una cesura entre historia y pasado análoga a la que le había criticado a de Certeau.

Una vez señalado el modo en que la articulación ricoeuriana entre representación mnémica e histórica impide la ruptura entre la escritura de la historia y el pasado, querría concluir estableciendo algunas pautas para repensar la crítica a de Certeau con respecto a la inaccesibilidad al pasado, aun teniendo presente que según este historiador el psicoanálisis se encuentra en condiciones de acceder a él. Considero que el énfasis de la crítica no debe recaer en la diferencia entre escritura histórica y pasado, donde uno la colocaría en una primera instancia, sino en la concepción de historia que manejan ambos autores. La incapacidad de la historia de acceder al pasado es, para de Certeau, más una denuncia que una

${ }^{27} \mathrm{MO}$, p. 502.

${ }^{28} \mathrm{MO}$, p. 502. 
descripción de un estado de cosas. La práctica historiográfica es legitimadora de las condiciones de producción existentes, aunque, paradójicamente, aborde temáticas que trastocan estas condiciones de producción, y que las demás ciencias dejaron de lado ${ }^{29}$. Justamente la homogeneización de lo otro es la vía utilizada para anular este componente subversivo. El psicoanálisis, por el contrario, se presentaría como un discurso alternativo que busca abrirse a través de los rastros de ficción que quedan en medio de la objetividad y cientificidad de la escritura histórica. Ricoeur, en cambio, no recoge el aspecto político de la práctica historiográfica, lo que significa, por una parte, que el presente no busca homogeneizar al pasado, y por la otra, que no habría semejante tensión entre historia y psicoanálisis. De hecho, la articulación del haber sido y el ya no más, proporcionaría una suerte fundamento ontológico para que el historiador haga las veces de analista de la memoria colectiva.

\section{Resumen}

El presente artículo compara el modo en que Ricoeur y de Certeau conciben la relación entre el escrito histórico y el pasado. Se sostiene que existe una importante modificación en el pensamiento de Ricoeur sobre esta cuestión en el período comprendido entre Tiempo y narración y La memoria, la bistoria, el olvido. En el primero, la articulación entre narración histórica y pasado se produce en la puesta en intriga mientras que la segunda vuelve a una concepción de la historia basada en el acontecimiento, cambio que acerca al filósofo al pensamiento de Certeau. Seguidamente, matizamos la crítica que el filósofo le realizara a de Certeau en Tiempo y narración acerca de su tesis acerca de que la narración histórica es distinta del pasado. Por último, concluimos justificando nuestra tesis de que el desarrollo de $L a$ memoria, la bistoria, el olvido logra salvarse de esta crítica gracias al uso que Ricoeur hace del concepto de representación.

Palabras-clave: Acontecimiento, diferencia, posibilidades, representación, psicoanálisis.

29 “Considerada a continuación como 'disciplina', la historiografía es una ciencia que no tiene los medios para serlo. Su discurso toma a su cargo lo que más resiste a la cientificidad (la relación social con el acontecimiento, con la violencia, con el pasado, con la muerte), es decir, lo que cada disciplina científica debió eliminar para constituirse" (HP, p. 74). 


\begin{abstract}
This paper studies the way in which Ricoeur and de Certeau conceives the relationship between historical narration and past. Its hypothesis is that there has been a change in Ricoeur's position between Time and Narrative and Memory, History and Oblivion. In the first book the articulation between historical narration and past is articulated by the plot. In the second one, the concept of event makes his analysis more similar to de Certeau's. We discuss Ricoeur's critic to de Certeau in Time and Narrative and sustain that Memory, History and Oblivion. is not affected by this critic thanks to the concept of representation.
\end{abstract}

Key words: Event, difference, possibilities, representation, psychoanalysis. 